\title{
OBSERVATIONS OF MOLECULES IN STELLAR ATMOSPHERES - CHEMISTRY NEAR THERMAL EQUILIBRIUM
}

\author{
David L. Lambert \\ Department of Astronomy \\ The University of Texas \\ Austin, TX 78712 USA
}

\begin{abstract}
A general review is given of the astrophysical information obtainable from observations of molecules in stellar photospheres. Through selected examples, the use of molecules as thermometers (e.g., the OH $3 \mu \mathrm{m} V-R$ lines in the Sun and $\alpha O r i$ ) and as probes of the isotopic (e.g., ${ }^{i} \mathrm{Mg}$ in metal-poor $d$ warfs, ${ }^{12} \mathrm{C} /{ }^{13} \mathrm{C}$ in cool carbon stars) and elemental äbundances (e.g., CNO in red giants) is sketched. All of the (carefully) selected analyses assume that local thermodynamic equilibrium (LTE) prevails.
\end{abstract}

\section{Introduction}

In this review, I concentrate on the observations of molecules in the spectra of stellar photospheres. Other contributors were invited to extend discussion out through the chromospheres and into the circumstellar shells. Discussions of stellar photospheres may generally assume that conditions approach those of Local Thermodynamic Equilibrium (LTE). To the purist, LTE may be tantamount to translating the subject matter from the domain of the chemist to that of the physicist. In my experience of analysing stellar spectra, one delves frequently into those books and journals housed in the chemistry library and unavailable in either the astronomy or physics libraries. If LTE may be assumed, the targets of ones library searches and conversations with chemists are such basic molecular data as line lists, molecular constants, f-values, and dissociation energies. On discarding the LTE approximation, one calls for theoretical and experimental information on a variety of collisional excitation, ionisation, and dissociation processes. One may predict that, in the next dedade, the growth of non-LTE studies of stellar atmospheres will be stimulated by continuing improvements in the quality of stellar spectra, the development of numerical techniques for handling radiative transfer in multilevel model molecules, and (I hope) the application of new theoretical and experimental techniques to the measurement of the collisional cross-sections involving (principally) $\mathrm{H}$, $\mathrm{H}_{2}$, and $\mathrm{He}$.

Molecules are observable in a wide range of stars. A high temperature boundary near the main sequence is set by the possible presence of molecules in some cool Ap stars. This boundary extended to lower luminosities encounters the $C$-rich white dwarfs whose spectra contain $\mathrm{C}_{2}$ and $\mathrm{CH}$ lines. The low temperature boundary is, of course, the Hayashi limit. Most of the current work on molecules in stellar atmospheres concerns the 
giants and supergiants of spectral types G, K, M, MS, S, and the C-rich types R and N. Very little work has been reported on molecules in the $\mathrm{K}$ and $\mathrm{M}$ dwarfs.

Recent growth in observational studies of molecules in stellar atmospheres has been stimulated by several factors:

- New theoretical and experimental techniques are providing vital basic data on molecular structure and spectra.

-- The infrared is now accessible to the stellar spectroscopist. One may justify the importance of the infrared by noting that (i) cool stars whose atmospheres are rich in molecules emit most of their flux in the infrared, (ii) the vibration-rotation bands of these molecules fall in the infrared, and (iii) infrared spectra of cool stars are, in general, easier to analyse than the visible spectra with their very high density of atomic and molecular lines.

- Red giants are testbeds for theories of stellar evolution and nucleosynthesis. Mixing between the hot interior and the cool extensive envelope is predicted to change the chemical composition of the observable atmosphere. Many of the elements affected by the mixing are observable as molecules; e.g., the CNO isotopes are rearranged by the CNOtricycles during $\mathrm{H}$ burning and, in the coolest giants and supergiants, their elemental and isotopic abundances must be extracted from the molecular lines because atomic lines are unavailable.

- Substantial advances have been made in the construction of model atmospheres for cool stars. Techniques now exist for handling the line blanketing arising from millions of molecular (and atomic) lines. For restricted cases, the standard geometrical assumption of plane-parallel layers has been discarded in favor of spherical layers. Many challenges remain including thorough investigations of non-LTE effects, the hydrodynamics of turbulent supergiants and pulsating Mira variables, the inadequacies in the basic molecular data. Whenever possible, a manufacturer of model atmospheres and his customers should engage in an iterative exchange of information.

\section{Unique Contributions by Molecules}

To the easily intimidated, an association of atoms into molecules results in a spectrum of awful complexity. However, on reflection, the complexity is seen to offer novel opportunities to gain valuable information about the structure and composition of the stellar atmosphere. My emphasis here is on that information readily provided by molecules, but obtainable only with difficulty from atoms. In this section, I provide general remarks on two topics concerning stellar atmospheres:

- the temperature profile.

- the chemical composition.

In $\S 3$, I try to illustrate how molecules are being employed in observational attacks on thermally pulsing stars on the asymptotic giant branch.

\subsection{The Temperature Profile}

Spectroscopists have long used the measured equivalent widths of lines of an atom (or ion) to derive an excitation temperature $\left(\mathrm{T}_{\text {exc }}\right)$ for that species. If $\mathrm{LTE}$ is valid, this $\mathrm{T}_{\text {exc }}$ is obviously a weighted mean of the kinetic temperature over the layers contributing to the lines' formation. In recent years, comparison of the measured $\mathrm{T}_{\text {exc }}$ and predictions from model atmospheres has been used to derive a star's effective temperature $\left(T_{\text {eff }}\right)$. Space prohibits a thorough critique of the atomic lines as a stellar thermometer. While valuable 
and valid estimates of $\mathrm{T}_{\text {eff }}$ are provided for many types of stars, there are often severe limitations to this application of atomic lines. A majority of the limitations are rooted in fundamentals of atomic structure and spectra.

Use of atomic or molecular lines as a thermometer providing $\mathrm{T}_{\mathrm{exc}}$ and, thence, $\mathrm{T}_{\text {eff }}$ requires that there be available an adequate collection of weak lines sampling a range in excitation potential, possessing accurately known gf-values, and drawn from levels whose populations show no significant departures from LTE. The latter constraint is cited because, with few exceptions predictions of non-LTE populations for atoms in stellar atmospheres have not advanced beyond a level that I call 'semi-quantitative' and do not permit the $\mathrm{T}_{\text {exc }}$ (NLTE)- $\mathrm{T}_{\text {eff }}$ relation to be predicted. This is especially true for cool stars and those atomic species frequently adopted as thermometers (e.g. Fe I, Ti I). My list of qualitative conditions could be translated to quantitative conditions embracing the number of lines, the range in excitation potential, the accuracy of the gf-values and the precision of the stellar equivalent widths.

My intent is not to explore the quantitative accuracy of $T_{\text {exc }}$-estimates, but to point out that molecules offer inherent advantages over atoms. Molecules are more practical thermometers than atoms. In particular, the rotational structure of a given band of an electronic or vibration-rotation transition provides a set of lines whose relative f-values may often be fixed to satisfactory accuracy from a rotational line strength (Hönl-London factor) and a quantity describing the vibration-rotation interaction. The range in excitation potential across the sample of lines, which controls the sensitivity of the thermometer, may be extended by combining bands provided that their relative $\mathrm{f}$-values are known. Preliminary theoretical studies indicate that the rate constants for rotational and vibrational excitation and deexcitation through $\mathrm{H}, \mathrm{He}, \mathrm{H}_{2}$ and e collisions dominate the statistical equilibrium of the ground and, possibly, the excited vibrational states of a molecule resident in the photosphere of a red giant (Thompson 1973; Hinkle and Lambert 1975); i.e., the local excitation and kinetic temperatures are identical or almost so.

By contrast, atomic spectra are far less attractive thermometers. An atomic line list spanning an adequate range in excitation potential will necessarily involve different terms which are most probably connected by strong permitted radiative transitions and, hence, subject to non-LTE effects. A satisfactory line list may also span a broader wavelength inverval than the molecular alternative. In general, the available supply of accurate atomic $\mathrm{f}$-values will be found wanting.

To illustrate the utility of a molecular thermometer, I sketch recent applications of the vibration rotation $(V-R)$ transitions of the hydroxyl $(\mathrm{OH})$ radical's ${ }^{2} \Pi$ ground state. This example also illustrates the potential of current $a b$ initio calculations in quantum chemistry. A literature search will reveal that there is no reliable laboratory measurement of the absolute f-value of the V-R lines; measurements of the relative f-values in the fundamental sequence are of an interesting accuracy and serve to constrain the absolute $f$ value - see Mies (1974). The f-values are calculable given the electric dipole moment function (EDMF- $\mu(\mathrm{R})$ where $\mathrm{R}$ is the internuclear separation), and the potential function. The most accurate theoretical prediction for the EDMF would appear to be that Werner $e t$ al. (1983). It predicts correctly the measured dipole moments for low rotational levels in the vibrational levels $\mathrm{v}=0,1$, and 2 (Peterson et al. 1984). In general, the RKR potential functions are to be preferred to the (similar) theoretical functions for the computation of the $V-R$ wavefunctions and, thence, the matrix element $\left\langle v^{\prime} J^{\prime}|\mu(R)| v^{\prime \prime} R^{\prime \prime}\right\rangle$ upon which the $f-$ value depends.

A thorough analysis of $\mathrm{OH}$ pure rotation and vibration-rotation transitions in the solar spectrum was provided bySauval et al. (1984) and Grevesse et al . (1984). Figure 1 shows how the solar photosphere may serve as a spectroscopic standard source and, also, 
how $\mathrm{OH}$ is a practical thermometer. Three $a b$ initio predictions of the EDMF and two solar model atmospheres were tested. A solution may be deemed satisfactory if the oxygen abundances (log $\varepsilon(\mathrm{OL})$ are both the same for all $\mathrm{OH}$ line and equal to that abundance provided by the atomic oxygen forbidden lines which appear to be reliable indicators of the oxygen abundance (Lambert 1978). Inspection of Figure 1 shows that there is such a

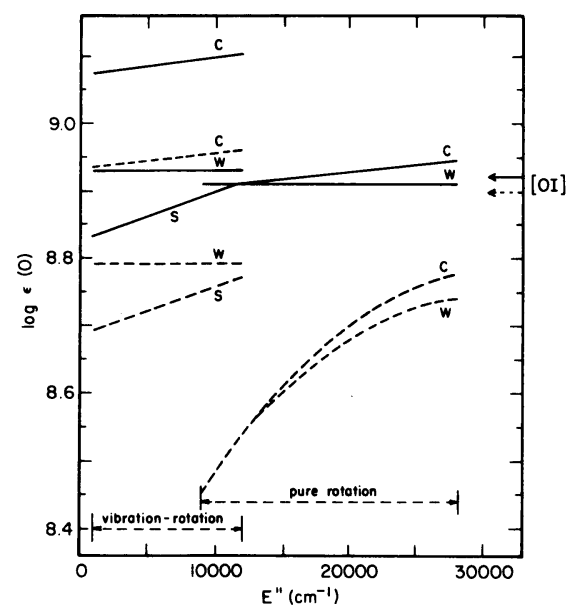

Fig. 1. The solar oxygen abundance as provided by $\mathrm{OH}$ pure rotation and vibrationrotation lines of various (lower) excitation potentials (E") - after Grevesse et al. (1984). The lines represent least-squares fits to results from individual lines for two model atmospheres (solid lines $\equiv$ Holweger-Müller 1974; dashed lines $\equiv$ Vernazza et al. 1976) and three ab initio EDMFs (C $\equiv$ Chu et al. 1974; $\mathrm{S} \equiv$ Stevens et al. 1974; W $\equiv$ Werner et al. 1983). The oxygen abundance given by the [O I] lines is marked by arrows on the right-hand side (Lambert 1978).

solution: the combination of Werner et al.'s EDMF and the Holweger-Müller (1974) model atmosphere. Although a mathematical purist might argue over the uniqueness of this result, I would note that external assessments of both the EDMF and the model favor the selection provided by Figure 1. Peterson et al'.s measurements of the electric dipole moment match predictions by Werner et al., but not those of other ab initio calculations. The solar photospheric brightness temperatures are reproduced well by the Holweger-Müller model, but significantly less satisfactorily by Vernazza et al.s model. Furthermore, analysis of the solar CO fundamental V-R lines shows clearly a similar failing of Vernazza et al."s model (Harris and Lambert 1986).

As a second application of the OH V-R lines, I sketch the results of an analysis of the fundamental lines near $3 \mu \mathrm{m}$ in the spectrum of the M supergiant $\alpha$ Ori (Lambert et al. 1986). In the $3 \mu \mathrm{m}$ window, the stellar line density is low. OH with its open band structure is essentially the sole contributor. Hence, after the telluric lines are ratioed out, accurate equivalent widths are available for many $\mathrm{OH}$ lines. Figure 2 shows a composite curve of growth constructed for a model atmosphere at $\mathrm{T}_{\text {eff }}=3800 \mathrm{~K}$ and $\mathrm{f}$-values based on Stevens et al.'s EDMF (see Mies 1974). In this case, $\mathrm{OH}$ proves not to be suitable as a thermometer. The available line selection is such that the equivalent width and excitation potential are well correlated. Since most of the lines are saturated, and the range in excitation potential at a given equivalent width is small, the $\mathrm{OH}$ lines are here a better 
indicator of the atmospheric microturbulence than the temperature. (On spectra of superior quality, it may be possible to detect weak satellite lines and to restore $\mathrm{OH}$ as a sensitive thermometer.) Two large departures from the predicted curve of growth are apparent: the strongest observed lines, all from the 1-0 and 2-1 bands, are stronger than expected, and the 5-4 lines fall off the predicted curve.

Location of the 5-4 lines is readily attributed to cancellation within the $<5 \mathrm{~J}^{\prime}|\mu(\mathrm{R})| 4 \mathrm{~J}^{\prime \prime}>$ matrix elements which is due, in large part, to the fact that the gradient of the EDMF changes sign at an internuclear separation only slightly larger than the equilibrium separation $\left(r_{e}\right)$. The $f$-values for 5-4 lines are extremely sensitive to the precise form of the EDMF. With the more recent EDMF by Werner $e$ t al., the offset of the 5-4 lines from the curve of growth defined by the other bands is greatly reduced. One senses an opportunity here to improve our knowledge of the OH EDMF through the analysis of stellar lines; 5-4 lines were not detected in the solar spectra analysed by Grevesse et al.

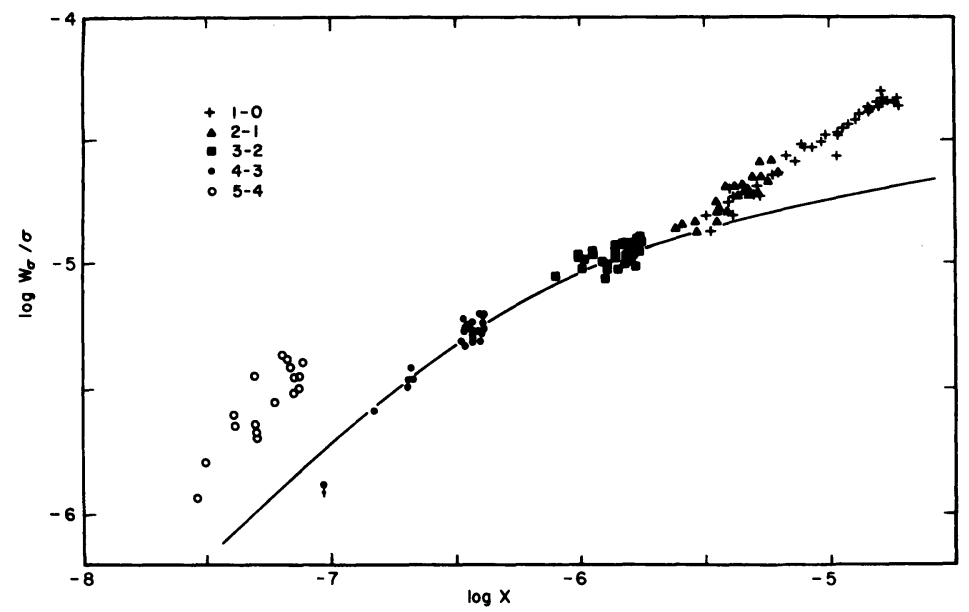

Fig. 2. A composite curve of growth for $\mathrm{OH}$ V-R lines in $\alpha$ Ori. The solid line is a theoretical curve of growth for a depth-independent microturbulence of $3 \mathrm{~km} \mathrm{~s}^{-1}$.

The systematic departure of the 1-0 and 2-1 lines from the predicted curve cannot be ascribed to errors in the adopted $f$-values. Several potential explanations may be entertained. The model atmosphere may be in error: line blanketing may have been represented incorrectly or the assumed plane parallel geometry may be inappropriate. Calculations show that the cores of these strong lines are formed at the top of the photosphere in layers that standard theoretical models cannot pretend to represent accurately; for example, deposition of mechanical energy and production of a chromosphere is most likely in these layers. In addition, departures from LTE may be possible in these outermost layers; the sense of the discrepancy is such that the line source function is smaller than the local Planck function. One looks forward to a fuller analysis of the $\mathrm{OH}$ (and other) lines.

Other potential thermometers in the infrared include the V-R bands of $\mathrm{CO}$ in both Oand $\mathrm{C}$-rich stars and of $\mathrm{SiO}$ in O-rich stars. The $\mathrm{CN}$ red system, an electronic transition, provides several band sequences of which the major part of the $\Delta v=-2$ sequence near 2 $\mu \mathrm{m}$ is free from serious bending with other molecular lines in both the $\mathrm{O}$ - and C-rich red giants. The $\Delta \mathrm{v}=-3$ sequence near $4 \mu \mathrm{m}$ is present at a useful strength in the cool carbon stars (Lambert et al. 1986, here LGEH). 


\subsection{The Chemical Composition}

Nothing more than an introduction to the molecules' contributions to the delicate exercise of extracting the chemical composition from a stellar spectrum is possible in the limited space available. An attempt is presented in the hope that a few converts may be found. I divide the subject matter into two parts:

- isotopic ratios.

- elemental ratios

\subsubsection{Isotopic Abundance Ratios}

Three concise statements suffice to outline why molecular lines in stellar spectra are analysed for the isotopic abundance ratio of one or more of the molecule's constituent atoms:

- Often, the isotopic ratios address astrophysical questions of great import, e.g., stellar nucleosynthesis of the chemical elements.

- Often, ratios of high accuracy are obtainable with limited knowledge of the atmospheric structure and a partial description of the basic molecular data, e.g., the ${ }^{12} \mathrm{C} /{ }^{13} \mathrm{C}$ ratio may be extracted from $\mathrm{CN}$ lines without a precise value for the molecule's dissociation energy whose persistent uncertainty afflicts all attempts to use the $\mathrm{CN}$ lines as monitors of either the $\mathrm{C}$ or the $\mathrm{N}$ elemental abundances.

- In general, the detectable molecules in stellar spectra provide isotopic ratios for those atoms for which the isotopic atomic wavelength shifts are too small to be resolved in stellar spectra. This fortunate circumstance arises because the nuclear masses have a direct influence on a molecule's V-R energy levels.

One final introductory note - a combination of isotopic and elemental abundance ratios is often essential in addressing the key astrophysical questions.

To illustrate these introductory comments and other selected aspects of isotopic abundance analyses, I discuss two recent studies:

- the ${ }^{24} \mathrm{Mg} / 25 \mathrm{Mg} / 26 \mathrm{Mg}$ ratios for metal-poor stars.

- the ${ }^{12} \mathrm{C} /{ }^{13} \mathrm{C}$ ratio in cool carbon stars.

The ${ }^{24} \mathrm{Mg} /{ }^{25} \mathrm{Mg} / 26 \mathrm{Mg}$ ratios. The atmospheric composition of a metal-poor unevolved star is assumed to be identical in all major respects to that of the interstellar cloud from which it formed; lithium is one of a few obvious exceptions to this assumption. It is through such metal-poor stars that the chemical evolution of the Galaxy is traced. Predictions of the nucleosynthesis achieved for both explosive (Arnett 1969) and hydrostatic burning (Woosley and Weaver 1982) of light fuels such as carbon, show that production of neutron-rich nuclei is inhibited in metal-poor mixtures; i.e., the isotopic abundance of ${ }^{25} \mathrm{Mg}$ and ${ }^{26} \mathrm{Mg}$ and, also, the elemental abundances of $\mathrm{Na}$ and $\mathrm{Al}$ (the sole stable nuclei are ${ }^{23} \mathrm{Na}$ and ${ }^{27} \mathrm{Al}$ ) are predicted to decline relative to ${ }^{24} \mathrm{Mg},{ }^{28} \mathrm{Si}$, and other so-called $\alpha$-nuclei. Both the $\mathrm{Na} / \mathrm{Mg}$ and $\mathrm{A} 1 / \mathrm{Mg}$ ratios are obtainable but, until very recently, published results by different authors lacked consistency. Therefore, pursuit of the prediction through the $\mathrm{MgH} \mathrm{A}^{2} \Pi-\mathrm{X}^{2} \Sigma$ transition and the $\mathrm{Mg}$ isotopic ratio was begun.

For the $\Delta \mathrm{v}=0$ sequence, which provides the strongest stellar lines, the isotopic wavelength shifts are small because the $\mathrm{A}$ and $\mathrm{X}$ states have similar vibrational and rotational constants and, hence, the weak ${ }^{25} \mathrm{MgH}$ and ${ }^{26} \mathrm{MgH}$ lines are blended with the stronger ${ }^{24} \mathrm{MgH}$ line, but detectable as an asymmetry of the stellar line profile. Extraction 
of the $\mathrm{Mg}$ isotopic ratio was achieved for sunspots (Sotirovski 1971) and $\mathrm{K}$ and $\mathrm{M}$ giants (Boesgaard 1968; Bell and Branch 1970; Tomkin and Lambert 1976, 1979). Our analysis (Tomkin and Lambert 1980) of $\mathrm{Gmb} 1830$, an unevolved cool dwarf with $[\mathrm{Fe} / \mathrm{H}]=-1.3$, provided an upper limit on the ${ }^{25} \mathrm{Mg}$ and ${ }^{26} \mathrm{Mg}$ (here, ${ }^{i} \mathrm{Mg}$ where $\mathrm{i}=25$ or 26 ) abundances which was less than the solar/terrestrial value: ${ }^{i} \mathrm{Mg} /{ }^{24} \mathrm{Mg}<7 \%$, but ${ }^{25} \mathrm{Mg} /{ }^{24} \mathrm{Mg}=$ $12.7 \%$, and ${ }^{26} \mathrm{Mg} /{ }^{24} \mathrm{Mg}=13.9 \%$ for terrestrial samples. Lambert and McWilliam (1986) - see Figure 3 -also obtain a low limit to ${ }^{\mathrm{i}} \mathrm{Mg} /{ }^{24} \mathrm{Mg}(\leq 3 \%)$ by an analysis of $v$ Ind, a subgiant with $[\mathrm{Fe} / \mathrm{H}]=-1.52$. Barbuy (1985) provides estimates of ${ }^{\mathrm{i}} \mathrm{Mg}$ for a selection of (mainly) metal-poor dwarfs and giants. McWilliam and Lambert (1986) rediscuss stars first analysed by Tomkin and Lambert (1980). In Figure 4, I show currently available results for ${ }^{\mathrm{i}} \mathrm{Mg} /{ }^{24} \mathrm{Mg}$ versus $[\mathrm{Fe} / \mathrm{H}]$ for dwarfs and subgiants. The abundance of the neutron-rich isotopes ${ }^{25} \mathrm{Mg}$ and ${ }^{26} \mathrm{Mg}$ declines with increasing metal-deficiency, a trend predicted by theories of stellar nucleosynthesis. The decline is more striking if ${ }^{1} \mathrm{Mg} /{ }^{24} \mathrm{Mg}$ is referenced to $[\mathrm{Mg} / \mathrm{H}] ;[\mathrm{Mg} / \mathrm{Fe}]>0$ in metal-poor stars. Additional isotopic analyses are desirable. These will call for high-resolution high S/N spectra of fainter stars: the $\mathrm{MgH}$ features are weak and, in many cases, blended with atomic $\mathrm{C}_{2}$ lines.

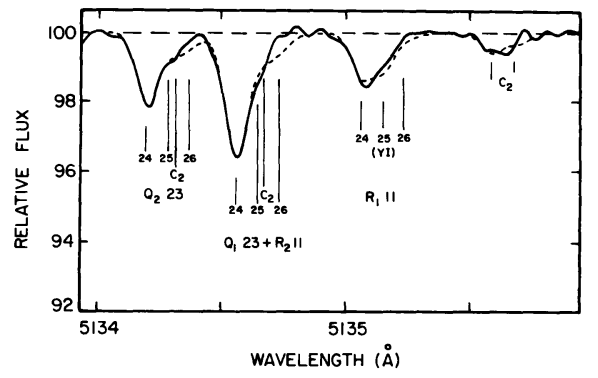

Fig. 3. Spectrum of $v$ Indi near $5135 \AA$. The observed spectrum is shown by the solid line. For the synthetic spectrum (dashed line), the $\mathrm{Mg}$ isotopes were given their terrestrial abundances and all known blending lines were included. Examination of the $\mathrm{MgH}$ profiles clearly shows that the isotopic $\mathrm{Mg}$ abundances in $v$ Indi are less than the terrestrial abundances.

A different astrophysical question may be answered by the $\mathrm{Mg}$ isotopic ratios: What is the neutron source for the s-processing seen in the atmospheres of red giants such as the $\mathrm{Ba}$ II, $\mathrm{S}$ and $\mathrm{N}$ stars? Two candidates meet some rather general criteria: ${ }^{22} \mathrm{Ne}(\alpha, n){ }^{25} \mathrm{Mg}$ and ${ }^{13} \mathrm{C}(\alpha, \mathrm{n})^{16} \mathrm{O}$ with the former operating at a higher temperature than the latter and, hence, it is favored as the source in intermediate mass stars. Low mass stars ( $\mathrm{m} \leqslant 3 \mathrm{M}_{\odot}$, perhaps) are supposed not to ignite ${ }^{22 \mathrm{Ne}}$ in the zones between the $\mathrm{He}$ and $\mathrm{H}$ burning shells, and, hence, ${ }^{13} \mathrm{C}(\alpha, \mathrm{n})$ is suspected to be their neutron source. One differentiating characteristic between s-processing driven by ${ }^{22} \mathrm{Ne}(\alpha, \mathrm{n})$ rather than ${ }^{13} \mathrm{C}(\alpha, \mathrm{n})$ is the high abundance expected for ${ }^{25} \mathrm{Mg}$ and ${ }^{26} \mathrm{Mg}$ from the former. Analysis of $\mathrm{MgH}$ lines in classical $\mathrm{Ba}$ II, one mild $\mathrm{Ba}$, and $2 \mathrm{MS}$ and $2 \mathrm{~S}$ stars show no significant enhancement of the heavier Mg isotopes (Tomkin and Lambert 1979;. Clegg, Lambert and Bell 1979; Smith and Lambert 1986). This and certain characteristics of the elemental 
abundances exclude ${ }^{22} \mathrm{Ne}(\alpha, \mathrm{n})$ as the primary neutron source. Our search for s-processed material synthesized by the ${ }^{22} \mathrm{Ne}$ source in (presumably) an intermediate mass star is continuing.

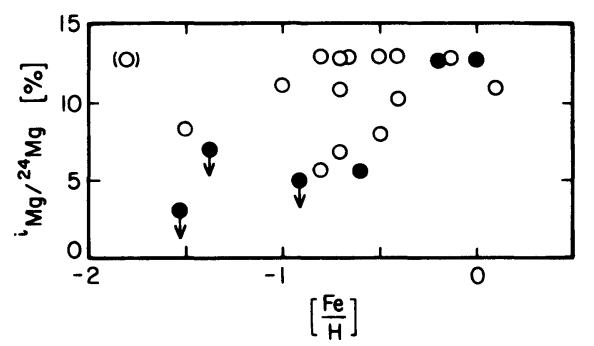

Fig. 4. $\mathrm{Mg}$ isotopic abundances as a function of metallicity. Observations (here ${ }^{\mathrm{i}} \mathrm{Mg}=$ $25 \mathrm{Mg}$ or ${ }^{26 \mathrm{Mg}}$ ) are taken from Barbuy (1985 - open circles) and Tomkin and Lambert (1980), Lambert and McWilliam (1986), and McWilliam and Lambert (1986) - solid symbols for the latter three references. The symbol in parentheses is based on a spectrum described as 'bad' by Barbuy.

The ${ }^{12} \mathrm{C} /{ }^{13} \mathrm{C}$ Ratio in Cool Carbon Stars. These stars and their $\mathrm{C}_{2}$ Swan system bands provided the first detection of ${ }^{13} \mathrm{C}$ in an extraterrestrial object, but the same characteristic that underlay this early discovery - namely, the great strength of the $\mathrm{C}_{2}$ Swan bands - bedevils attempts to obtain an accurate estimate of the ${ }^{12} \mathrm{C} /{ }^{13} \mathrm{C}$ ratio. The same comments applies to the CN Red system lines in the visible and near-infrared which have proven a popular source of a ${ }^{12} \mathrm{C} /{ }^{13} \mathrm{C}$ ratio. A compilation of published ratios would bewilder the reader unfamiliar with the spectra - see the summary provided by Lambert (1980).

Since ${ }^{13} \mathrm{C}$ is linked to the evolutionary origins of the carbon stars, estimates of the ${ }^{12} \mathrm{C} /{ }^{13} \mathrm{C}$ ratio continue to be published. Here, I review our novel analyses using highresolution infrared spectra (see LGEH). Today, theoretical studies of AGB stars show that ${ }^{12} \mathrm{C}$ from the He-burning shell may be mixed into the stellar envelope and, thence, into the atmosphere. In the likely event that this mixing is achieved without significant exposure to the $\mathrm{H}$-burning CNO-cycles, the ${ }^{12} \mathrm{C} /{ }^{13} \mathrm{C}$ ratio of a carbon star is predicted to increase approximately linearly with the ${ }^{12} \mathrm{C} /{ }^{16} \mathrm{O}$ ratio and to be rather directly related to the lower ${ }^{12} \mathrm{C} /{ }^{13} \mathrm{C}$ and ${ }^{12} \mathrm{C} /{ }^{16} \mathrm{O}$ ratios seen in the $\mathrm{O}$-rich $\mathrm{M}$ giants, the obvious progenitors of carbon stars. There remains a possibility that deep exposures of oxygen-rich or carbon-rich gas to the CNO-cycles may have driven the $\mathrm{C} / \mathrm{O}$ ratio to equilibrium values in excess of unity. In this event, the resulting carbon star is necessarily $\mathrm{N}$-rich and ${ }^{13} \mathrm{C}$-rich with ${ }^{12} \mathrm{C} /{ }^{13} \mathrm{C} \sim 3.4$, the equilibrium value. This scenario may possibly occur in very luminous AGB stars on development of a deep convective envelope with a very hot base.

Visible and near-infrared spectra of carbon stars are so crowded with strong lines that the location of the continuum level is uncertain and weak lines are rare. These spectroscopic facts of life surely account for the discrepancies between observers who analyse spectra of similar quality. In selected intervals of the infrared, the line density is lower and several favorable opportunities exist for a measurement of the ${ }^{12} \mathrm{C} /{ }^{13} \mathrm{C}$ ratio. 
LGEH exploited the CN Red system $\Delta v=-2$ lines near $2 \mu \mathrm{m}$ and the CO V-R $\Delta v=2$ and 3 lines.

Figure 5 shows a narrow interval of the $\mathrm{CN} \Delta \mathrm{v}=-2$ sequence. The continuum is definable with fair precision from the numerous narrow windows across $500 \mathrm{~cm}^{-1}$. Weak ${ }^{12} \mathrm{CN}$ lines are identifiable; most are satellite lines and a few are high rotational members of the main $(\mathrm{P}, \mathrm{Q}, \mathrm{R})$ branches. In the typical carbon star, weak ${ }^{13} \mathrm{CN}$ lines are from the main branches present in large numbers. The difference in $\mathrm{f}$-values between the main and satellite lines is such that for a ${ }^{12} \mathrm{C} /{ }^{13} \mathrm{C}$ ratio of about 20 to 40 , the satellite ${ }^{12} \mathrm{CN}$ and typical ${ }^{13} \mathrm{CN}$ line have comparable (and small) equivalent widths. Since the lines also have similar excitation potential, the derived ${ }^{12} \mathrm{C} /{ }^{13} \mathrm{C}$ ratio is insensitive to the adopted excitation or effective temperature. The advantages of combining satellite ${ }^{12} \mathrm{CN}$ and main ${ }^{13} \mathrm{CN}$ lines was noted first by Fujita and his colleagues in analyses of near-infrared $\mathrm{CN}$ $(\Delta v=+2)$ lines - see, for example, Fujita and Tsuji (1977).

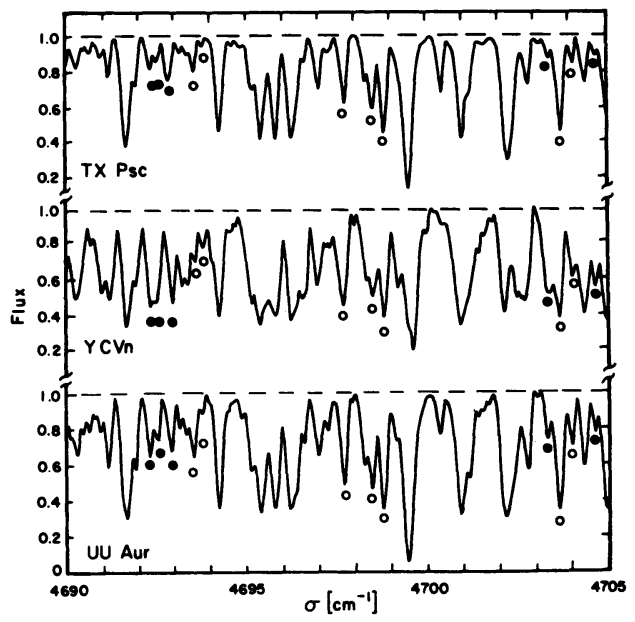

Fig.5 A portion of the spectrum of the cool carbon stars TX Psc, Y CVn, and UU Aur. The majority of the lines are attributable to the ${ }^{12} \mathrm{CN}$ and ${ }^{13} \mathrm{CN}$ molecules. The open circles identify a few ${ }^{12} \mathrm{CN}$ lines. Note the pair near $4704 \mathrm{~cm}^{-1}$ : the stronger line is $0-2$ $\mathrm{Q} 48$ and the weaker line is the satellite $\mathrm{O}_{\mathrm{P}_{12}} 48$. The filled circles identify a number of ${ }^{13} \mathrm{CN}$ lines. Note the great strength of these lines in the ${ }^{13} \mathrm{C}$-rich star $\mathrm{Y} \mathrm{CVn}$.

LGEH describe two methods of extracting the ${ }^{12} \mathrm{C} /{ }^{13} \mathrm{C}$ ratio from the CO V-R lines. The $\Delta \mathrm{v}=3$ bands near $1.6 \mu \mathrm{m}$ provide weak ${ }^{13} \mathrm{CO}$ lines. A comparison of weak ${ }^{12} \mathrm{CO}$ and ${ }^{13} \mathrm{CO}$ lines within this sequence necessarily pairs high excitation ${ }^{12} \mathrm{CO}$ and lower excitation ${ }^{13} \mathrm{CO}$ lines and, hence, the ${ }^{12} \mathrm{C} /{ }^{13} \mathrm{C}$ ratio is dependent on the temperatures in the line-forming region; i.e., the effective temperature and the chemical composition which, through the line blanketing, influences the temperature profile. An alternative scheme combines the ${ }^{13} \mathrm{CO}$ lines from the stronger (i.e., larger $\mathrm{f}$-value) $\Delta \mathrm{v}=2$ bands near $2.5 \mu \mathrm{m}$ with the ${ }^{12} \mathrm{CO}$ lines at $1.6 \mu \mathrm{m}$, and then, as in the $\mathrm{CN}$ case, ${ }^{12} \mathrm{CO}$ and ${ }^{13} \mathrm{CO}$ lines of similar excitation potential are compared and the sensitivity of the ${ }^{12} \mathrm{C} /{ }^{13} \mathrm{C}$ ratio to atmospheric structure is slight. These three methods yield similar results. 
The histogram of the ${ }^{12} \mathrm{C} /{ }^{13} \mathrm{C}$ ratios for our 30 cool carbon stars (Figure 6) shows that the typical star has a low ${ }^{13} \mathrm{C}$-content. Examination of the ${ }^{12} \mathrm{C} /{ }^{13} \mathrm{C}$ and the ${ }^{12} \mathrm{C} /{ }^{16} \mathrm{O}$ ratios shows that these carbon stars may have been produced by the addition of nearly pure ${ }^{12} \mathrm{C}$ to the envelope of a $\mathrm{M}$ giant, i.e.

$$
\frac{\left({ }^{12} \mathrm{C} /{ }^{13} \mathrm{C}\right)_{\mathrm{C}}}{\left({ }^{12} \mathrm{C} /{ }^{13} \mathrm{C}\right)_{\mathrm{M}}} \sim \frac{\left({ }^{12} \mathrm{C} /{ }^{16} \mathrm{O}\right)_{\mathrm{C}}}{\left({ }^{12} \mathrm{C} /{ }^{16} \mathrm{O}\right)_{\mathrm{M}}}
$$

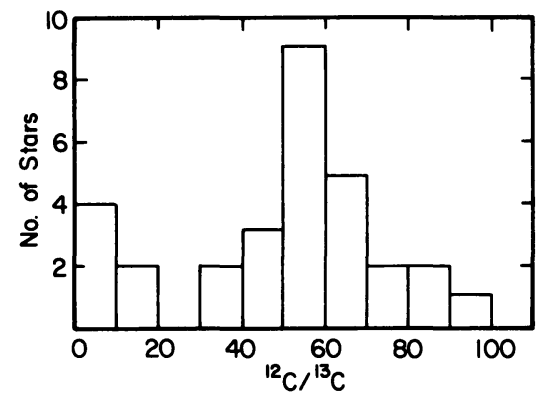

Fig.6 Histogram of the ${ }^{12} \mathrm{C} /{ }^{13} \mathrm{C}$ ratios for 30 cool carbon stars.

Our new results certainly confirm well-founded suggestions by Fujita and his colleagues that carbon stars have, in general, a low ${ }^{13} \mathrm{C}$ content. On the other hand, our infrared spectra confirm many earlier claims that ${ }^{13} \mathrm{C}$-rich carbon stars exist; for example, for RY Dra, T Lyr, Y CVn, the ${ }^{12} \mathrm{C} /{ }^{13} \mathrm{C}$ ratios (3.6, 3.2, and 3.5, respectively) are not significantly different from the predicted ratio (3.4) for the CNO cycle in equilibrium. A fourth star commonly put with the above trio is WZ Cas, but its ratio, ${ }^{12} \mathrm{C} /{ }^{13} \mathrm{C}=4.5$, is distinctly above this predicted value.

\subsubsection{Elemental Abundance Ratios}

In quantitative analyses of warm/solar stars, molecular spectra may supplement the data on elemental abundance ratios provided by atomic spectra. However, in the cooler stars such as the inhabitants of the AGB, molecular spectra may be the primary or even the sole source of elemental abundance ratios. Certainly, for the CNO trio whose abundances reflect the presence of $\mathrm{H}$ and $\mathrm{He}$ burning products in the stellar atmosphere, molecules are the key to the abundance, as Table 1 may illustrate. This Table, which is not intended to be comprehensive, is drawn from the several published analyses cited in the footnotes. Classification of the lines as 'primary' or 'secondary' reflects their status as assumed by the authors. One may anticipate that some classifications may be revised as either new experiments and calculations provide more precise data on the molecules or fresh opportunities for acquiring stellar spectra are seized; for example, high-resolution infrared spectra of $\mathrm{G}$ and $\mathrm{K}$ giants would yield $\mathrm{CO}, \mathrm{OH}$, and $\mathrm{CN}$ lines to complement or to replace the assortment of atomic and molecular visible and near-infrared lines previously used.

Note too that a simultaneous analysis of two or more indicators may be required for a single elemental abundance. Consider, for example, the carbon stars. Carbon 
monoxide and $\mathrm{N}_{2}$ largely control the partial pressures of free $\mathrm{C}, \mathrm{N}$, and $\mathrm{O}$ and, hence, of molecules containing these elements. While the $\mathrm{CO}$ lines are a direct measure of the $\mathrm{O}$ abundance $\varepsilon(O)$, the $C_{2}$ lines reflect the difference $(\varepsilon(C)-\varepsilon(O))^{2}$ and, hence, the $C$ abundance is set by the combination of $\mathrm{CO}$ and $\mathrm{C}_{2}$ lines. An obvious extension of this to $\mathrm{CN}$ shows that the $\mathrm{N}$ abundance requires a simultaneous fit to $\mathrm{CO}, \mathrm{C}_{2}$, and $\mathrm{CN}$ lines. $\mathrm{A}$ corollary is obvious: errors in the basic data (f-values and dissociation energies) for a molecule may affect the abundance of more than one element and the effects may not be equal. For example, an error in the $\mathrm{f}$-value of the $\mathrm{C}_{2}$ lines contributes the following errors:

$$
\begin{aligned}
& \Delta \log (\varepsilon(C)-\varepsilon(O)) \sim 0.5 \Delta \log f\left(C_{2}\right) \\
& \Delta \log \varepsilon(N) \sim \Delta \log f\left(C_{2}\right)
\end{aligned}
$$

where the $\mathrm{CN}$ lines are the source of the $\mathrm{N}$ abundance and 'all' of the $\mathrm{N}$ is supposed to be associated into $\mathrm{N}_{2}$ (i.e., $\mathrm{p}\left(\mathrm{N}_{2}\right) \propto \varepsilon(\mathrm{N})$ and $\mathrm{p}(\mathrm{N})^{2} \propto \mathrm{p}\left(\mathrm{N}_{2}\right)$, hence $\varepsilon(\mathrm{N}) \propto \mathrm{p}(\mathrm{CN})^{2} ; \mathrm{p}=$ partial pressure).

Derived abundances are subject to error from directions other than the basic molecular data. Reasonably thorough assessment of the total errors are now a fairly common feature of published analyses. Space prohibits discussion of this topic. However, as a measure of precision now possible, I show in Figure 7 histograms of the oxygen abundances for four samples:

- G and K giants analysed by Lambert and Ries (1981) using the [OI] ( and the $\mathrm{C}_{2}$ Swan) lines,

- M, MS, and S stars from Smith and Lambert $(1985,1986)$ who considered the $\mathrm{OH}$ V-R (and CO V-R) lines,

- cool carbon stars from LGEH who used the CO V-R lines,

- C-rich planetary nebulae from Aller and Czyzak (1983) who, of course, analyse the nebular emission lines.

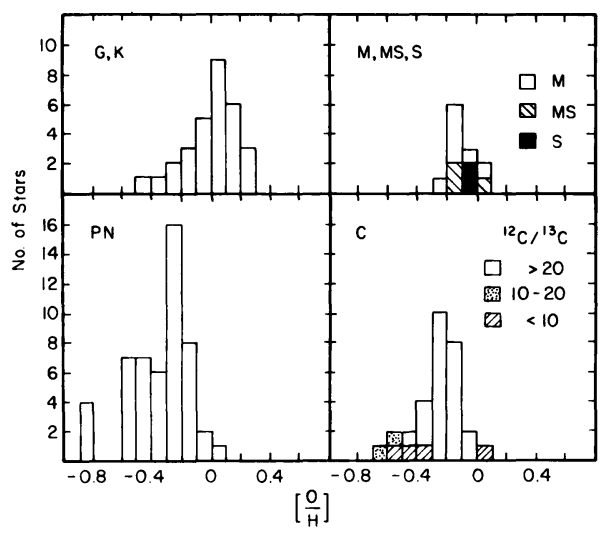

Fig.7 Histograms of [O/H] for four samples: $\mathrm{G}$ and $\mathrm{K}$ giants (Lambert and Ries 1981); M, MS, and S stars (Smith and Lambert 1985, 1986); C-rich planetary nebulae (Aller and Czyzak 1983); cool carbon stars (LGEH - see key to the ${ }^{12} \mathrm{C} /{ }^{13} \mathrm{C}$ ratios). 
Since the ages of the samples must be very similar and the oxygen abundances are not predicted to be affected significantly by internal nucleosynthesis and mixing, one may reasonably suppose that all samples should have very similar oxygen abundances. Inspection of Figure 7 shows that this expectation is met. The $\mathrm{O}$ abundance in the $\mathrm{G}$ and $\mathrm{K}$ giants appears to be systematically higher than in the other samples; this reflects, in part, the high adopted $\mathrm{T}_{\text {eff }}$ 's; a lower and more conventional $\mathrm{T}_{\text {eff }}$ scale would give lower $\mathrm{O}$ abundances - see Kjaeergaard et al. (1982). Although the overall consistency is most pleasing, complacency is not to be encouraged because consistency may, in part, result from a conspiracy between two or more sources of error. Certainly, other elements show annoying differences from sample to sample; for example, the $\mathrm{N}$ abundances for cool carbon stars are less than those reported for the $G, K$, and $M$ giants - see LGEH for a discussion.

TABLE 1

Indicators of CNO Abundances in Red Giants

\begin{tabular}{llll}
\hline $\begin{array}{l}\text { Spectral } \\
\text { Type }\end{array}$ & Primary Lines & Secondary Lines & Refs. \\
\hline G and $\mathrm{K}$ & $\mathrm{C}_{2}$ Swan $\Delta v=0,-1$ & CH A-X $\Delta v=0,-1$ & LR,K,G \\
& CN Red $\Delta v=4$ to 2 & CN Red $\Delta v=-1,-2$ & \\
& {$[\mathrm{OI}$ 6300 and 6363 $\mathrm{A}$} & CN Violet $\Delta v=0,-1$ & \\
& & CO V-R $\Delta v=3$ & \\
& & OH V-R $\Delta v=2$
\end{tabular}

$\begin{array}{ll}\text { M, MS, S } & \text { CO V-R } \Delta v=3,2 \\ & \text { OH V-R } \Delta v=2,1 \\ & \text { NH V-R } \Delta v=1 \\ & \text { CN Red } \Delta v=-2 \\ \text { SC, C } & \text { CO V-R } \Delta v=3,2 \\ & \text { C } 2 \text { Phillips } \Delta v=-2 \\ & \text { CN Red } \Delta v=-2\end{array}$

$\mathrm{C}_{2}$ Ballik-Ramsay

DWS

$\mathrm{CH}$ V-R $\Delta \mathbf{v}=1$

LGEH

NH V-R $\Delta v=2$

$\mathrm{HCN}$ V-R $\Delta \mathbf{v}=1$

References:

DWS = Dominy, Wallerstein, and Suntzeff (1986)

$\mathrm{G}=$ Gratton (1985)

LGEH = Lambert et al. (1986)

$\mathrm{SL}=$ Smith and Lambert $(1985,1986)$

K Kjaeergaard et al. (1982)

LR = Lambert and Ries (1981)

In closing this section, I offer a golden rule: all atomic and molecular lines pertinent to the abundance of the element in question should be analysed. Adherence to this rule is difficult, but dangerous to nothing except ones false sense of achievement. In the next section, I comment on two applications of the rule in our analysis of the cool carbon stars. 


\section{Departures from LTE - Two False Alarms?}

Our 1.5-2.5 $\mu \mathrm{m}$ spectra of the cool carbon stars provide lines of $\mathrm{C}_{2}, \mathrm{CO}$ and $\mathrm{CN}$ which are just sufficient to provide the $\mathrm{C}, \mathrm{N}$, and $\mathrm{O}$ abundances. Several additional molecules offer an opportunity to check the abundances. Calculations (Goorvitch et al. 1980) using model atmospheres suggest that the $\mathrm{H}_{2}$ quadrupole V-R lines should be present in spectra of the carbon stars. Examination of spectra shows that the lines, if present at all, are substantially weaker than predicted (Goorvitch et al. 1980; Johnson et al. 1983). Comparisons of predicted and observed emergent flux distributions around the $\mathrm{H}^{-} 1.6 \mu \mathrm{m}$ opacity minimum show that the observed flux peak is apparently much smaller than expected. These two signatures of the $\mathrm{H}$ content have led to the sugggestion that the atmospheres are H-deficient. Since the theoretical and observational ramifications of this suggestion are considerable, we explored more mundane explanations for the two discrepancies.

Archival spectra at $4 \mu \mathrm{m}$ for five carbon stars provide lines of the CN Red $\Delta \mathrm{v}=-3$ sequence, as well as the $\mathrm{NH}$ and $\mathrm{CH} \mathrm{V}-\mathrm{R}$ transitions from the ground electronic states. Our model atmospheres and CNO abundances account satisfactorily for the observed strengths of the $4 \mu \mathrm{m} \mathrm{CN}$ and $\mathrm{NH}$ lines. To our dismay, the observed $\mathrm{CH}$ lines are weaker than predicted. This discrepancy occasioned a thorough search for an explanation.

In the following sections, I sketch our unsuccessful attempts to attribute both the unexpected weakness of the $\mathrm{H}_{2}$ and the $\mathrm{CH}$ lines to departures from LTE. Although I comment on the true probable cause of the lines' small strengths, the reader is referred to LGEH for a full discussion.

\subsection{Are Carbon Stars H-deficient?}

An apparent deficiency of $\mathrm{H}^{-}$and $\mathrm{H}_{2}$ may result from non-LTE effects in the statistical equilibrium of the $\mathrm{H}^{-}$ion. The initial effect enters in the p.hotodetachment $\mathrm{H}^{-}+$photon $\rightarrow$ $\mathrm{H}+\mathrm{e}$ where the rate is set by the local mean intensity $\left(\mathrm{J}_{\mathrm{v}}\right)$ for $\lambda<16400 \AA$. Since, in the upper photosphere, $\mathrm{J}_{\mathrm{v}}$ exceeds the local Planck function $\left(\mathrm{B}_{\mathrm{v}}\right)$, the local density of $\mathrm{H}^{-}$ions is expected to be less than the LTE value. In turn, $\mathrm{H}_{2}$ is influenced because its formation is likely to be controlled by associative detachment. $\mathrm{H}+\mathrm{H}^{-} \Leftrightarrow \mathrm{H}_{2}+\mathrm{e}$.

Self-consistent non-LTE models incorporating the statistical equilibrium of $\mathrm{H}^{-}$and radiative equilibrium show that the surface layers are heated (relative to the comparable LTE model). The underabundance of $\mathrm{H}^{-}$and $\mathrm{H}_{2}$ is insufficient to account for the weak $\mathrm{H}_{2}$ lines and the absence of the $1.6 \mu \mathrm{m}$ flux peak; the $\mathrm{H}_{2}$ equivalent width is reduced by no more than 0.2 dex, but the magnitude of the discrepancy noted by Johnson et al. (1983) exceeds 1.0 dex.

Clearly, these non-LTE effects are not the main culprit. Although a fully consistent solution has not yet been found, the key to both problems is the line opacity contributed by the polyatomic molecules $\left(\mathrm{HCN}, \mathrm{C}_{2} \mathrm{H}_{2}\right)$. In discussion of the $\mathrm{H}_{2}$ lines and the $1.6 \mu \mathrm{m}$ flux peak, Johnson and colleagues refer to model atmospheres to which only diatomic molecules contribute line opacity. When the polyatomics' contribution is included, the atmosphere is back-warmed sufficiently to reduce the equilibrium abundance of $\mathrm{H}_{2}$ (Eriksson et al. 1984); LGEH show that the predicted and observed strengths of $\mathrm{H}_{2}$ lines are in fair agreement such that they are not evidence of a H-deficiency. Moreover, the predicted flux curve including the contributions overtone bands of the polyatomics and 
the changed atmospheric structure accounts fairly well for the apparent absence of the 1.6 $\mu \mathrm{m}$ peak. In short, there is no decisive evidence for a substantial $\mathrm{H}$ deficiency in the atmosphere of cool carbon stars.

\subsection{The CH V-R Lines}

In our (LGEH) analysis of the $1.5-2.5 \mu \mathrm{m}$ spectra of carbon stars, the available molecules - $\mathrm{CO}, \mathrm{CN}, \mathrm{C}_{2}$ - suffice to determine the $\mathrm{CNO}$ abundances. Apart from the $\mathrm{H}_{2}$ line's indirect dependence on these abundances through the molecules' line opacity, one must search other spectral windows for checks on the abundances. The $4 \mu \mathrm{m}$ window offers the $\mathrm{CH}$ and NH V-R transitions from the electronic ground states as well as the $\Delta \mathrm{v}=-3$ sequence of the CN Red system.

On examination of spectra of five stars, LGEH were dismayed to find that the $\mathrm{CH}$ $\mathrm{V}-\mathrm{R}$ lines were observed to be weaker than predicted. Despite a survey of numerous potential sources of error and uncertainty, no viable explanation was found. This survey included quantitative exploration of non-LTE effects in the excitation and formation of $\mathrm{CH}$. In the former, we examined the possibility that the vibrational levels of the ${ }^{2} \Pi$ ground state could be populated via radiative transitions to and from the excited electronic states, e.g., $X^{2} \Pi\left(v^{\prime \prime}\right) \rightarrow B^{2} \Sigma^{-}\left(v^{\prime}\right) \rightarrow X^{2} \Pi\left(v^{\prime \prime} \pm n\right)$. At the wavelengths of these electronic transitions, the mean intensity exceeds the local Planck function and, hence, the vibrational excitation of the ${ }^{2} \Pi$ state exceeds the local kinetic temperature. However, even when ameliorating collisional processes are omitted, these radiative processes are insufficient to reduce the predicted $\mathrm{CH}$ intensities to the observed level. This failure is attributable to the fact that the $\mathrm{CH}$ lines are formed deep in the photosphere where $\mathrm{J}_{\mathrm{v}} \sim \mathrm{B}_{\mathrm{v}}$. A similar calculation invoking photodissociation as the primary destruction mechanism of $\mathrm{CH}$ fails for the same reason.

LGEH dispose of other potential explanations: errors in the adopted f-values or the molecular (LTE) abundances, a $\mathrm{H}$ deficiency.There is a possibility that the $4 \mu \mathrm{m}$ photospheric spectrum is diluted by radiation from circumstellar dust; LGEH tentatively discarded this idea because the strengths of the $\mathrm{CN} 4 \mu \mathrm{m}$ lines were predicted correctly from the $2 \mu \mathrm{m}$ lines. Another possibility is that the adopted effective temperatures are systematically too cool. They are based upon angular diameter from lunar occultations. If the star were embedded in a thick dust shell, the occultation would measure the diameter of the shell rather than the photosphere. Adoption of this larger diameter results in an effective temperature that is too cool. However, a revision of the temperature scale by the 500 to $1000 \mathrm{~K}$ necessary to account for the observed $\mathrm{CH}$ lines may introduce other inconsistencies. An attempt is underway to measure precise excitation temperatures for $\mathrm{CO}$ and $\mathrm{CN}$ in order to check the temperature scale.

\section{Concluding Remarks}

As long as LTE is an adequate assumption, the basic molecular data required by astrophysicists is restricted to analyses of the spectra (e.g., wavelength, excitation potential), dissociation energies of the observed molecules' and others controlling the partial pressures of the constituent atoms, and to the $f$-values of the observed lines and of those transitions contributing to the molecular line blanketing. While much data of high quality exist, 'shopping lists' can be provided on request!

This review has paid scant attention to the chemical processes occurring in a stellar atmosphere. As long as LTE is an adequate assumption, detailed information on rates of 
chemical reactions and internal excitations and deexcitations is not required. On further examination, it may well turn out that non-LTE effects are more important than now appear to be the case for the stars discussed here. In part, the apparent lack of interest reflects the omission of topics in stellar atmospheres to which non-LTE effects may be endemic: e.g., the development and propagation of a shock through the photosphere of a pulsating star such as a Mira variable; the structure of the chromosphere and the circumstellar shell of a cool star with a wind. It is also the case that information on the molecular processes is so far from complete that theoretical studies of non-LTE effects can be no more than exploratory, e.g., the internal excitation of the vibronic levels of a molecule $\mathrm{AB}$ is controlled in large part by inelastic collisions with $\mathrm{H}$ and $\mathrm{He}$ atoms, $\mathrm{H}_{2}$ molecules and, perhaps, electrons. In all but the coolest stars, $\mathrm{H}^{-}$atoms so outnumber $\mathrm{H}_{2}$ molecules that $\mathrm{AB}+\mathrm{H}$ collisions are likely to dominate the internal excitation of $\mathrm{AB}$ (Hinkle and Lambert 1975). Unfortunately, rather little is known about the cross-sections for vibrational and rotational excitation of astrophysical molecules by $\mathrm{H}$ atoms.

This brief review will have satisfied a personal goal if the sketches of stellar evolution and nucleosynthesis stimulate experimental and theoretical chemists to explore those molecules of astrophysical interest, and if stellar spectroscopists are persuaded to hunt in the literature and the laboratories of chemical physics for the basic data so essential to our understanding of the structure and chemical composition of stellar atmospheres. Although my selection of topics concentrated on molecules and their spectra, atomic lines are carriers too of information. Often, a combination of atomic and molecular lines must be assembled in order to address astrophysical questions.

I thank the editors for their patience. Since the particular problems sketched here are largely drawn from collaborative analyses, I am indebted to several colleagues (Drs. K. Eriksson, B. Gustafsson, K. H. Hinkle, V. V. Smith, and Mr. A. McWilliam.) My research in stellar spectroscopy, evolution and nucleosynthesis is supported in part by the National Science Foundation (grant AST 83-16635) and the Robert A. Welch Foundation.

\section{REFERENCES}

Aller, L. H., and Czyzak, S. J. 1983, Ap. J. Suppl., 51, 211.

Arnett, W. D. 1969, Ap. J. 157, 1369.

Barbuy, B. 1985, Astr. Ap., 151, 189.

Bell, R. A., and Branch, D. 1970, Ap. J. (Letters), 5, 203.

Boesgaard, A. M. 1968, Ap. J., 154, 185.

Chu, S. I., Yoshimine, M., and Liu, B. 1974, J. Chem. Phys., 61, 5389.

Clegg, R. E. S., Lambert, D. L., and Bell, R. A. 1979, Ap. J., 234, 188.

Dominy, J. F., Wallerstein, G., and Surtzeff, N. B. 1986, Ap. J., 300, 325.

Eriksson, K., Gustafsson, B., Jørgensen, U. G., and Nordlund, Ä 1984, Astgr. Ap., $132,37$.

Fujita, Y., and Tsuji, T. 1977, P.A.S. Japan, 29, 711.

Gratton, R. G. 1985, Astr. Ap., 148, 105.

Goorvitch, D., Goebel, J. H., Augason, G. C. 1980, Ap. J., $240,588$.

Grevesse, N., Sauval, A. J., and van Dishoeck, E. F. 1984, Astr. Ap., 141, 10.

Harris, M. J., and Lambert, D. L. 1986, in preparation.

Hinkle, K. H., and Lambert, D. L. 1975, M.N.R.A.S., 170, 447.

Holweger, H., and Müller, E. A. 1974, Solar Phys., 39, 19.

Johnson, H. R., Goebel, J. H., Goorvitch, D., and Ridgway, S. T. 1983, Ap. J. (Letters), 270, L63. 
Kjaeergaard, P., Gustafsson, B., Walker, G. A. H., and Hultqvist, L. 1982, Astr. Ap., $115,145$.

Lambert, D. L. 1978, M.N.R.A.S, 82, 249. - 1980, in "Les Spectres des Molecules Simples au Laboratoire et en Astrophysique", XXIe Coll. Int. D'Ast., Liège, p. 173, 1980.

Lambert, D. L., Brown, J. A., Hinkle, K. H., and Johnson, H. R. 1984, Ap. J., 284, 223.

Lambert, D. L., Gustafsson, B., Eriksson, K., and Hinkle, K. H. 1986, Ap. J. Suppl. in press (LGEH).

Lambert, D. L., and Ries, L. M. 1981, Ap. J., 248, 228.

Lambert, D. L., and McWilliam, A. 1986, Ap. J., in press.

McWilliam, A., and Lambert, D. L. 1986, in preparation.

Mies, F. H. 1974, J. Mol. Spec., 53, 150.

Peterson, K. I., Fraser, G. T., and Klemperer, W. 1984, Can. J. Phys., 62, 1502.

Sauval, A. J., Grevesse, N., Brault, J. W., Stokes, G. M., and Zander, R. 1984, Ap. J., $282,330$.

Smith, V. V., and Lambert, D. L. 1985, Ap. J., 294, 326.

Sotirovski, P. 1971, Astr. Ap., 14, 319.

Stevens, W. J., Das, G., Wahl, A. C., Krauss, M., Neumann, D. 1974, J. Chem. Phys., 61, 3686.

Thompson, R. I. 1973, Ap. J., 181, 1039.

Tomkin, J., and Lambert, D. L. 1976, Ap. J., 208, 436. . 1979, Ap. J., 227, 209. 1980, Ap. J., 235, 925.

Vernazza, J. E., Avrett, E. H., and Loeser, R. 1976, Ap. J. Suppl., 30, 1.

Werner, H. J., Rosmus, P., and Reinsch, E. A. 1983, J. Chem. Phys., 79, 905.

Woosley, S. E., and Weaver, A. 1982, in Essays in Nuclear Astrophysics, ed. C. A. Barnes, D. D. Clayton, and D. N. Schramm (Cambridge: CUP), p. 377. 